plications in adults, in whom it has been associated with a seronegative arthropathy, ${ }^{5}$ which may or may not be accompanied by a rash. It may also produce aplastic crises in patients with chronic haemolytic anaemias such as hereditary spherocytosis and sickle cell disease. ${ }^{6}$

Practitioners concerned in antenatal care should therefore be alert to the possibility of parvovirus infection and its possible complications. Women complaining of a rash or arthropathy during pregnancy or who are in contact with a child with erythema infectiosum should be screened serologically for parvovirus infection. If infection is confirmed the fetus should be monitored by maternal serum $\alpha$ fetoprotein concentrations, which may predict fetal infection, and by ultrasound to detect fetal hydrops. Fetal blood sampling may also be of value in diagnosing fetal anaemia. Parvovirus is an appreciable cause of non-immune fetal hydrops and resultant fetal loss and women at risk should be screened for evidence of infections during pregnancy.

I A GREER

Centre for Reproductive Biology,

University of Edinburgh,

Edinburgh EH3 $9 \mathrm{EW}$

1 Mortimer PP, Cohen BJ, Buckley MM, et al. Human parvovirus and the fetus. Lancet 1985;ii: 1012 .

2 Anand A, Gray ES, Brown T, Clewley JP, Cohen BJ. Human parvovirus infection in pregnancy and hydrops fetlis. $N$ Engl. Med 1987;316:183-6.

3 Knott PF, Welply GAC, Anderson MJ. Serologically proved intrauterine infection with parvovirus. Br Med $\mathcal{F} 1984 ; 289$. 1660.

4 Carrington D, Gilmore DH, Whittle MJ, et al. Maternal serum alphafetoprotein-a marker of fetal aplastic crises during
intrauterine human parvovirus infection. Lancet 1987;i:433-5. 5 Schneider AP, Rauhaus AP. Human parvovirus infection and seronegative like arthritis in adults. Lancet 1988;i:296.
seide

6 Rao KRP, Patel AR, Anderson MJ, Hodgson J, Jones SE Patison JR. Infection with parvovirus like virus and aplastic crisis in chronic hemolytic anemia. Ann Intern Med 1983;98: 930-2.

\section{Cough and angiotensin converting enzyme inhibitors}

SIR,-For Dr Christine Bucknall and others $(9$ January, p 86) to say that cough associated with converting enzyme inhibitors may be a variant of the cough in asthma may cloud the issue of the pathogenesis of this adverse reaction. Our epidemiological data and the observations of others show that there is a significant preponderance of women in reports of cough with captopril and enalapril. ${ }^{1-3}$ There is, however, no such sex difference with asthma, which suggests that the mechanism of production of cough with asthma and that with converting enzyme inhibitors are different. Unfortunately, the authors' study did not match controls and cases for sex, three of eight cases and six of nine controls being men.

The question of an association of angiotensin converting enzyme inhibitors with an asthma wheeze is interesting. Dr Bucknall and colleagues included in their study two patients with bronchial asthma who had reported increased wheeze after treatment with enalapril. These patients showed poor control of their asthma before the start of the rechallenge protocol, which started six and 12 weeks after they had stopped taking enalapril, and inhaled steroids had been introduced to control the increased wheeze. Our observations with the cough reaction were that symptoms cleared rapidly on withdrawal, the mean recovery time being 3.4 days for captopril and 5.5 days for enalapril. ${ }^{1}$ These facts would suggest that either the exacerbation of wheeze and the cough reaction were produced by different mechanisms or the wheeze was not a reaction and represented a natural progression of bronchial asthma.

We are not convinced that angiotensin convert- ing enzyme inhibitors include among their reactions exacerbation of bronchial asthma. The number of reports of cough so far received at this centre is 167 , but we have only eight reports of wheeze or exacerbation of asthma, and we have described three asthmatic patients with cough but no increase in wheeze. ${ }^{1}$ One study of seven patients with a cough attributable to converting enzyme inhibitors failed to show either reversible airflow obstruction or altered bronchial reactivity to methacholine. ${ }^{4}$ Prostaglandins might have a role in the pathogenesis of the cough reaction, ${ }^{1}$ and the observation that sulindac can clear or reduce the cough suggests that their contribution is a major one. ${ }^{5}$ This again highlights the difference from bronchial asthma, which is not alleviated by nonsteroidal anti-inflammatory drugs.

The incidence of a cough reaction is difficult to assess. Retrospective analyses of highly selected groups of patients-for example, those attending hypertension clinics — could produce an artificially high incidence. The incidence observed by us of $1 \cdot 1 \%$ for captopril and $2 \cdot 8 \%$ for enalapril ${ }^{1}$ is probably low because these were reported cases only, but the patient population was unselected and we believe the monitoring method used revealed most clinically important cases. The incidence of cough is probably close to these figures.

DAVID M COULTER I RALPH EDWARDS

New Zealand Medicines Adverse Reactions Committee, National Toxicology Group,

University of Otago Medical School

Dunedin, New Zealand

1 Coulter DM, Edwards IR. Cough associated with captopril and enalapril. BrMed f 1987;294:1521-3.

2 Webb D, Benjamin N, Collier J, Robinson B. Enalapril-induced cough. Lancet 1986;ii: 1094 .

3 Inman WHW. Enalapril-induced cough. Lancet 1986;ii: 1218.

4 Town GI, Hallwright GP, Maling TJB, O'Donnell TV. Angiotensin converting enzyme inhibitors and cough. $N Z \mathrm{Med} \mathcal{f}$ 1987;100: 161-3.

5 Nicholls MG, Gilchrist NL. Sulindac and cough induced by converting enzyme inhibitors. Lancet 1987;ii: 872.

\section{Skin reactions and fever with indapamide}

SIR,-We appreciate the interest that Drs D Kandela and D Guez (20 February, p 573) have shown in our short report (21 November, p 1313), but we find their letter misleading.

Our paper was a reviewed article and also follows the guidelines formulated by a workshop held under the auspices of Ciba-Geigy. ${ }^{1}$ Unfortunately the references of skin reactions to indapamide that they mention do not satisfy these criteria. Even so, we wonder why they did not include them in their product datasheet.

Although we clearly stated that our figures were based on data obtained by voluntary reporting, and that no conclusions could be drawn about the comparative incidence of adverse effects to the different drugs mentioned in the article, Drs Kandela and Guez have wrongly understood that it was our purpose to do so. Their statement about the "highly comparable absolute numbers of cases: 16 and 15 respectively" (of skin reactions to indapamide and chlorthalidone) is irrelevant: chlorthalidone is more widely used in The Netherlands than indapamide, and the relative number of skin reactions to indapamide was much higher than that to chlorthalidone. The same applies to their interpretation of the figures on (hydro) chlorothiazide and frusemide. There is no logic in comparing absolute numbers without comparing prescription figures, and we have some difficulties in understanding their concept "absolute incidence."

Our conclusion than indapamide can cause skin reactions, sometimes serious, still holds, and these reactions were not mentioned in the datasheet. (The reporting of skin reactions to indapamide has increased considerably during the past year, and the World Health Organisation database now holds 266 such reports.)

B H CH STRICKER

Netherlands Centre for Monitoring of

Adverse Reactions to Drugs,

2280 HK Rijswijk

The Netherlands

World Health Organisation Collaborating Centre

C BIRIELI.

for International Drug Monitoring,

Uppsala,

Sweden

\section{HIV, hepatitis B, and sexual behaviour}

SIR,-The report by Dr Brian A Evans and others (13 February, p 473) raises disturbing ethical issues, and I was surprised to see no reference to these. Screening for antibodies to the human immunodeficiency virus (HIV) has been the subject of much debate both in your journal and elsewhere.

They state that 1115 women were included in their study and that all were offered the opportunity to ask for an HIV antibody test; 207 accepted the offer, but this means that 908 did not. Surely this means that they were implicitly refusing consent for the test. Despite this the test was carried out on all women.

While the screening test was carried out "independently of and separate from the clinical records," the results were clearly traceable to individual patients as in the case of the one positive result they obtained. This patient was then again counselled. It is difficult to see how they explained this repeat counselling to her, alone of 1115 women, without raising anxiety that there was something wrong. The counsellor presumably knew the result of the first test and would have been encouraging the patient to be tested. Despite this she explicitly declined the test.

Thus the doctors were left with a highly important test result for which permission had been refused. What are they then to do with the result? It has been connected to her case notes. How can the patient be protected from accidental revelation of the result in the future? Is it ethical to withhold the information from the patient when it is critically important to her sexual and reproductive behaviour and her subsequent clinical management?

These impossible dilemmas could have been avoided if patients had not been tested when consent had been withheld.

Alex Mills

London NW5 INA

SIR,-Dr Brian A Evans and colleagues indicate a low prevalence of antibodies to the human immunodeficiency virus (HIV) in a large cohort of heterosexual women attending a sexually transmitted disease clinic in London. This may at first be reassuring, and the authors conclude that heterosexual women are at low risk in London, but the risk of drawing this conclusion from inadequate, or at times absent, information is perilous.

Those women who were found to be infected were sexual contacts of high risk individuals, and the reasonable conclusion must be that the majority who remained uninfected had sexual partners who were not infected. Transmission was not shown because there was no risk. All this study shows, therefore, is a low prevalence in intravenous drug users and bisexual men-and we know this 
already. It does not, however, mean that transmission will not occur when these conditions change, as they have in some parts of the United Kingdom, ${ }^{1}$ and it gives us no clues about the likely factors which operate in centres where heterosexual spread is being observed regularly.

This study provides no evidence of the partners' antibody state and is therefore not a study of heterosexual spread of HIV infection. Furthermore, the partners' risk factor was known in only $97(8 \cdot 7 \%)$ of the 1115 women studied. All it can do is draw tentative conclusions about local prevalence. The use of hepatitis $B$ antibody as a surrogate marker for HIV risk is of little value. Most cohorts of drug users and homosexual men have a very high prevalence of this marker but currently low prevalence of HIV antibodies. In these high risk groups its predictive value is limited, as exposure to this infection has often occurred over many years; the position with HIV is different.

The authors suggest that educational campaigns have been unsuccessful but admit that their prevalence study was carried out immediately afte the national media coverage. Behaviour change is complicated and not merely instantly responsive to any propaganda. There is considerable information emerging that the population does now understand the facts. Changes in behaviour will occur when adequate data, good data, suggest to the individual that he or she personally is at high risk. ${ }^{2}$ Papers like this run the risk of reassuring the population of their low risk state even when the authors' own evidence indicates the presence of the start of heterosexual problem. Our own paper and the work of others indicate that such complacency will soon be shown to be misguided as infectivity is better understood. Population studies showing any seropositivity at this juncture can only be interpreted as a problem. The assumption that the risk to the heterosexual population is low is the very reason why a long term outlook may be bleak. Behaviour in this group will change only when the risk is perceived as being high; unfortunately by that time the epidemic may suddenly have become obvious and unstoppable.

A J FRANCE

CAROL SKIDMORE

J R ROBERTSON

J J K ROBERTS

Edinburgh Drug Addiction Study, Edinburgh EH4 4PL

1 France AJ, Skidmore CA, Robertson JR, et al. Heterosexua spread of HIV infection in Edinburgh. Br Med $\mathcal{J}$ 1988;296: 526-9.

2 Robertson JR, SKidmore CA, Roberts JJK. HIV infection in intravenous drug users: a follow up study indicating changes in risk taking behaviour. $B r \mathcal{F}$ Addict (in press).

Authors' REPLY,-We hope that Dr Mills and others will be reassured to learn that to date no harm has resulted to any of the women in our study screened for antibodies to HIV without their explicit consent. Our patients were tested routinely without being asked and without any record being made in their clinical notes so that the fears expressed about implicit refusal, repeat counselling, and accidental revelation were eliminated by the study design. With regard to the one patient who was unexpectedly positive a second sample would have been essential anyway before the diagnosis could have been made, and this was refused.

It is difficult to follow the arguments in the letter from Dr France and his colleagues in Edinburgh. Their paper found HIV infection in seven $(5 \cdot 7 \%)$ of 123 people at risk by heterosexual intercourse with partners "classified as abuser of intravenous drugs, prostitute, or other." All seven found to be positive had been the regular partners of intravenous drug abusers for 18 months or more and knew that their partners were positive. Casual encounters in 39 subjects produced no one who was positive for antibodies to HIV.

A screening study of sexually active women became a priority after the government's decision to accept advice that HIV was a risk to the genera community and to proceed with this message in a national health education campaign. A study re stricted by consent might have found a simila prevalence to the one we reported but the rea prevalence could have been much higher and the study therefore of little value. Moreover, it seemed unethical to design a study which at the same time did not allow a woman infected by HIV to know if she so wished.

We have continued testing since this study but on the basis of informed consent, with a refusal rate of around $5 \%$, unrelated to risk behaviour. The prevalence of HIV infection has remained below $0.25 \%$, with four out of a further 1842 women positive in 1987. All of them had known of thei high risk as sexual partners of intravenous dru users or bisexual men. Our conclusion that there is no evidence of spread by casual vaginal intercourse is thus sustained and now further supported by th study from Edinburgh.

Finally, we wonder whether it is ethical to confront an individual at no known risk with the possibility of an incurable infection, particularly when knowledge of having had or been offered an HIV test, regardless of the reason, might prove prejudicial both personally and financially. May this not be the main reason why some patient decline testing?

\section{B A Evans} S G M MCCORMACK

Department of Genitourinary Medicine,

West London Hospital,

London W6 7DQ

SIR,-Dr Brian A Evans and others (13 February, p 473) conclude that heterosexual women in London are at a low risk of becoming infected with the human immunodeficiency virus (HIV). We did a similar survey in our department and suppor their conclusion.

Before the public information campaign abou HIV infection 730 women who attended this department as new patients were tested for antibodies to HIV virus and to hepatitis B core antigen All patients were fully counselled and the test were performed with informed consent. Fifty five patients had declined the test after counselling and were excluded.

Two women were positive for HIV. One had been a drug addict and the other came from central Africa. Neither was hepatitis B positive. One woman was positive for hepatitis $B$ antigen but was negative for HIV antibody. She had no known risk factors for hepatitis B. We do not yet know the hepatitis B state of her consort.

In an initial study 91 out of 355 patients had sexually transmitted diseases; the incidence of these diseases in women attending the clinic is high compared with the low incidence of HIV and hepatitis B infections.

We have not seen any HIV positive white heterosexuals other than from the recognised risk groups. We suspect that earlier fears of rapid spread of HIV infection among heterosexuals were exaggerated.

K SIVAKUMAR D D COELHO R BASU ROY

Department of Genitourinary Medicine Royal Victoria Hospital Bournemouth BH7 6JF

\section{Chickenpox in pregnancy}

SIR,-Drs K Boyd and E Walker (6 February, p 393) concluded that chickenpox in pregnancy is sufficiently hazardous to merit admission to hospital and early treatment with acyclovir. I support this recommendation and report a recent maternal death due to infection with varicella zoster.

A 28 year old woman was admitted in labour 31 weeks into her fifth pregnancy. Two of her children had recently had chickenpox. The typical maculovesicular rash appeared three days before admission, when she had a temperature of $39^{\circ} \mathrm{C}$. Symptoms and signs of a viral pneumonitis were present.

A boy weighing $1650 \mathrm{~g}$ was delivered vaginally in good condition. He was given zoster immune globulin, and treatment with acyclovir was started. $\mathrm{He}$ developed respiratory distress syndrome, bacterial pneumonitis, and septicaemia, but there was no clinical or serological evidence of varicella zoster infection. He was discharged at the age of 3 months.

After delivery the patient's condition deteriorated rapidly with clinical and radiological evidence of worsening pneumonitis. Treatment with acyclovir was started but before the patient could be transferred to the intensive care unit for ventilation she had a hypoxic cardiac arrest. Resuscitative measures were initially successful but cardiac and renal failure ensued and were complicated by coagulopathy. Despite peritoneal dialysis the patient died 11 days after delivery.

A necropsy was not performed but varicella zoster infection was confirmed by viral culture and antibody titre.

Varicella zoster infection in pregnancy, especially when complicated by pneumonitis, has a serious morbidity and mortality for mother and fetus. A maternal mortality of $40 \%$ has been reported. ${ }^{\prime}$ The outcome may be improved by better awareness of the implications of the condition in pregnancy, early aggressive use of antiviral chemotherapy, and early recourse to ventilatory support. ${ }^{2}$

R G WhITE

Royal Maternity Hospital,

Grosvenor Road, Belfast BT12 6BB

1 Chodos WS. Varicella in pregnancy: report of case and review of literature. 7 Am Osteopath Assoc 1982;81:644-6.

2 Landsberger EJ, Hager WD, Grossman III JH. Successful management of varicella pneumonia complicating pregnancy. A report of three cases. $\mathcal{F}$ Reprod Med 1986;31:311-4.

\section{How does smoking harm the duodenum?}

SIR,-Dr M Guslandi (30 January, p 311) reviews and supports the case for the use of "cytoprotective" agents for the treatment of duodenal ulcer in smokers, but these claims are based on questionable evidence.

Firstly, some of the quoted studies of the effect of smoking on ulcer healing have been criticised in terms of study design. ${ }^{1}$ More importantly, the evidence for differential healing in smokers is based on only one trial for each agent quoted, whereas there are other possible trials of these agents from which to draw. Most trials of healing agents in duodenal ulcer have not been stratified prospectively or have not been of sufficient size to enable accurate analysis of smoking as a secondary end point.

To shed some light on the smoking controversy we undertook a formal meta-analysis of the effect of smoking in trials of duodenal ulcer healing. Thirty trials representing 4451 patients and 64 individual study arms met the inclusion criteria of randomised double blind endoscopically controlled 